\title{
Evolutionary Models for Ultracool Dwarfs
}

\author{
Catarina S. Fernandes (10 ${ }^{1,2}$, Valérie Van Grootel ${ }^{1}$ (i), Sébastian J. A. J. Salmon ${ }^{1}$, Bernhard Aringer ${ }^{3,4}$, Adam J. Burgasser ${ }^{5}$ (D), \\ Richard Scuflaire $^{1}$, Pierre Brassard ${ }^{6}$, and Gilles Fontaine ${ }^{6}$ (i) \\ ${ }^{1}$ Space Sciences, Technologies and Astrophysics Research (STAR) Institute, Université de Liège, 19C Allée du 6 Août, B-4000 Liège, Belgium \\ c.fernandes@uliege.be \\ ${ }^{2}$ Niels Bohr Institute, University of Copenhagen, Øster Voldgade 5, DK-1350 Copenhagen, Denmark \\ ${ }^{3}$ Dipartimento di Fisica e Astronomia Galileo Galilei, Università di Padova, Vicolo dell'Osservatorio 3, I-35122 Padova, Italy \\ ${ }^{4}$ Observatorio Astronomico di Padova-INAF, Vicolo dell'Osservatorio 5, I-35122 Padova, Italy \\ ${ }^{5}$ Center for Astrophysics and Space Science, University of California San Diego, La Jolla, CA, 92093, USA \\ ${ }^{6}$ Département de Physique, Université de Montréal, Montréal, Québec H3C 3J7, Canada \\ Received 2018 December 22; revised 2019 April 26; accepted 2019 May 16; published 2019 July 10
}

\begin{abstract}
Ultracool dwarfs (UCDs) have emerged as key targets for searches of transiting exoplanets. Precise estimates of the host parameters (including mass, age, and radius) are fundamental to constraining the physical properties of orbiting exoplanets. We have extended our evolutionary code Code Liégeois d'Evolution Stellaire to the UCD regime. We include relevant equations of state (EOSs) for $\mathrm{H}, \mathrm{He}$, as well as $\mathrm{C}$ and $\mathrm{O}$ elements to cover the temperature-density regime of UCD interiors. For various metallicities, we couple the interior models to two sets of model atmospheres as surface boundary conditions. We show that including $\mathrm{C}$ and $\mathrm{O}$ in the EOS has a significant effect close to the H-burning limit mass. The typical systematic error associated with uncertainties in input physics in evolutionary models is $\sim 0.0005 \mathrm{M}_{\odot}$. We test model results against observations for objects whose parameters have been determined from independent techniques. We are able to reproduce dynamical mass measurements of LSPM J1314+1320AB within $1 \sigma$ with the condition of varying the metallicity (determined from calibrations) up to $2.5 \sigma$. For GJ $65 \mathrm{AB}$, a $2 \sigma$ agreement is obtained between individual masses from differential astrometry and those from evolutionary models. We provide tables of UCD models for various masses and metallicities that can be used as reference when estimating parameters for ultracool objects.
\end{abstract}

Key words: stars: late-type - stars: low-mass

\section{Introduction}

Ultracool dwarfs (UCDs) are cool, small, and dim objects lying at the faint, red end of the Hertzsprung-Russell (HR) diagram (M7 and cooler; Kirkpatrick et al. 1995), at the limit of core H-burning. UCD classifications encompasses the very low-masses stars and brown dwarfs (BDs). As these objects emit primarily in the near-infrared (NIR) and have low intrinsic luminosities, it took the development of efficient photometric detectors and spectrographs at NIR wavelengths in the late 1990s to populate the UCD region in the HR diagram (see Section 2.2 in Reid \& Hawley 2005 for a historical review). Today, it is known that UCDs represent at least $15 \%$ of the population of star-like objects in the solar neighborhood (see, e.g., Bartlett et al. 2017, and the RECONS collaboration ${ }^{7}$ ).

In parallel with the increased observational access to UCDs, theoretical evolutionary models have improved in the past decades. Key advances include D’Antona \& Mazzitelli (1996), who generated models for metal-poor stars, Burrows et al. (1993, 1997, the "Tucson group"), who included grain opacities in atmosphere models, and (Baraffe et al. 1995, 1998, the "Lyon group") who coupled interior models to full model atmospheres as boundary conditions. The most recent and commonly used model set is Baraffe et al. (2015; hereafter, BHAC15), who presented updated evolutionary models for low-mass stars and young BDs that use boundary conditions from BT-Settl model atmospheres (Allard et al. 2012a, 2012b; Rajpurohit et al. 2013, 2018b). These atmospheres include updated molecular opacity linelists and cloud formation, as well as atmospheric convection parameters

\footnotetext{
See www.recons.org.
}

calibrated with $2 \mathrm{D} / 3 \mathrm{D}$ radiative hydrodynamic simulations (Freytag et al. 2010, 2012). BHAC15 models also adopt the solar composition of Asplund et al. (2009), supplemented with abundances from Caffau et al. (2011) for C, N, O, Ne, P, S, K, Fe, $\mathrm{Eu}, \mathrm{Hf}, \mathrm{Os}$, and Th. The BHAC15 models provide a significant improvement over the Baraffe et al. (1998) models when comparing to observations. In particular, the weaknesses in the Baraffe et al. (1998) color-magnitude diagrams (e.g., for the optical $(V-I)$ colors that were too blue for a given magnitude; and for the NIR colors for relatively old objects) were significantly improved by BHAC15.

The BHAC15 grid is publicly available and is widely used when trying to retrieve the parameters of UCDs (e.g., age, mass) from observations. This grid is given for solar composition with stellar mass from $1.40 M_{\odot}$ down to $0.01 \mathrm{M}_{\odot}$, with the lowest $T_{\text {eff }}=1200 \mathrm{~K}$ for $0.01 \mathrm{M}_{\odot}$, which corresponds to an age of $41 \mathrm{Myr}$. At low effective temperatures, the limit of validity of evolutionary models is set by the limit of validity of model atmospheres (Saumon \& Marley 2008). These models are likely robust for $T_{\text {eff }} \gtrsim 2000 \mathrm{~K}$, as below this temperature there are significant uncertainties associated with cloud formation and condensation of chemical compounds, which become important opacity sources in the atmosphere (Tsuji et al. 1996; Marley \& Leggett 2009; Morley et al. 2012).

In this paper, we present adaptations made to our in-house evolutionary code Code Liégeois d'Evolution Stellaire (CLES; Scuflaire et al. 2008) to produce UCD models: relevant equations of state (EOSs) suitable for the low-temperature and high-density regime, and relevant model atmospheres used as boundary conditions for the interior. The main motivation behind this work is the SPECULOOS project (Search for Planets Eclipsing 
Ultra-cool Stars; Gillon 2017; Delrez et al. 2018), a survey searching for transiting planets in the habitable zone of the nearest and brightest UCDs. The prototype of the SPECULOOS project is the TRAPPIST telescope (TRAnsiting Planet and PlanetesImals Small Telescope; Gillon et al. 2011), which led to the discovery of seven Earth-sized planets transiting the UCD TRAPPIST-1 (Gillon et al. 2016, 2017). More generally, transiting surveys are now focused on very cool stars, because of their small radii with close-in habitable zones. Atmospheric characterization of these exoplanets will be within reach of the next-generation telescopes (e.g., James Webb Space Telescope, European Extreme Large Telescope). In this context, precise estimates of the mass, radius, luminosity, effective temperature, and age for a host star are important to thoroughly characterize its exoplanets (see, for TRAPPIST-1, Van Grootel et al. 2018).

This paper is organized as follows. Section 2 provides the details of our CLES evolutionary models for UCDs, general properties and systematic error estimates, and a comparison to BHAC15 UCD models. Section 3 gives a series of test cases comparing models to observations. Section 4 presents our conclusions.

\section{CLES Models for UCDs}

\subsection{Input Physics}

The CLES evolutionary code was developed in the early 2000 s by the asteroseismology group of the University of Liège, and has been continuously updated. We present here only the main input physics used for UCDs, and refer to Scuflaire et al. (2008) for the main numerical features (see also Buldgen et al. 2016, 2017a, 2017b, for CLES in the context of the Sun and solar-like stars). CLES includes different choices for EOS, opacity, and atmosphere tables but here we mention only those relevant for the study of UCDs.

We considered $\mathrm{H}, \mathrm{He}, \mathrm{C}$, and $\mathrm{O}$ for the EOSs of UCD objects. We directly adapted tables built for white dwarfs and subdwarf B stars (Brassard \& Fontaine 1994). These tables cover a large domain of the temperature-density plane $(2.10 \leqslant \log T \leqslant 8.98$ and $-12.0 \leqslant \log \rho \leqslant 9.0$ ), which includes UCDs at all evolutionary stages. Four EOS tables are available, one for each element considered. For each table, three regimes are invoked. First, for the low-density region, a network of Saha equations is solved for a mixture of radiation and an almost ideal (including Coulomb corrections), non-degenerate, partially or fully ionized gas composed of a mixture of $\mathrm{H}, \mathrm{He}, \mathrm{C}$, and $\mathrm{O}$ in various proportions. Second, in the partial ionization region where nonideal and electron degeneracy effects are important (intermediate densities), we used the EOS of Saumon et al. (1995) for H and $\mathrm{He}$, an improved version of the EOS of Fontaine et al. (1977) for $\mathrm{C}$ and for $\mathrm{O}$. Third, the high-density domain corresponds to the fully ionized plasma in liquid and ultimately solid phases, according to the physics described in Lamb (1974) and improved by Kitsikis et al. (2005). The low-density boundary of the second regime matches very smoothly with the high-density boundary of the first regime, thus ensuring that there are no significant jumps in the thermodynamic variables of interest. The connection between the high-density boundary of the partial degeneracy regime with the low-density boundary of the totally ionized domain is made at the location where the electron degeneracy parameter $\eta$ is equal to 20. Care has been taken to ensure that thermodynamic consistency is respected as explained in Fontaine et al. (1977). Interpolation in composition is handled following the additive volume prescription of Fontaine et al. (1977). This additive volume prescription is the best viable option for handling mixture in the EOSs of individual elements (Vorberger et al. 2007; Wang et al. 2013; Danel \& Kazandjian 2015). The FreeEOS equation of state $^{8}$ (Irwin 2012) version 2.2.1. (EOS1 configuration) is also available in CLES. It calculates the EOS without radiation pressure for specified mixtures using an efficient free-energy minimization technique. However, this did not converge for the lowest temperatures/highest densities encountered in $\mathrm{H}$-burning stars below $\sim 0.14 \mathrm{M}_{\odot}$ at solar composition.

Model atmospheres based on $T(\tau)$ relations (including gray atmospheres) are inaccurate as boundary conditions (BCs) for interior models of UCDs (Chabrier \& Baraffe 1997). We have implemented in CLES two sets of surface boundary conditions (density, temperature, geometrical depth) from detailed model atmospheres. The first derives from the publicly available BTSettl model atmospheres (Allard et al. 2012a, 2012b). The second makes use of the model atmospheres of B. Aringer, originally developed for asymptotic and red giant branch stars (Aringer et al. 2016, hereafter, AR16). In both cases, the transition between interior and atmosphere is performed at the Rosseland mean optical depth $\tau=100$ (similar to the BHAC15 models), as a safe limit to avoid discrepancies at the boundary while treating convection and adiabatic processes (Chabrier \& Baraffe 1997). For BT-Settl model atmospheres and interior structure, we used the models computed with the solar abundances of Asplund et al. (2009), as for the interior structure, and followed the same heavy-element abundance as for the Sun (i.e., $[\alpha / H]=0$ ). This results in four distinct solarscaled compositions: $[M / H]=-0.5,0.0,+0.3,+0.5$. The AR16 models are computed for Asplund et al. (2009) solar (and meteoritic when available) abundances as well, supplemented by Caffau et al. (2011) for C, N, O, Ne, and Ar. Seven compositions are possible, from $[M / H]=-2$ to 1 , by steps of 0.5. AR16 is computed with the COMARCS program, which is based on the version of the MARCS code of Gustafsson et al. (2008), and uses the Copenhagen Opacities for Model Atmospheres opacity generation code by Aringer (2000). Unlike the BT-Settl models, AR16 models are dust-free and thus limited to $T_{\text {eff }} \geqslant 2600 \mathrm{~K}$.

CLES includes opacities from the OPAL (Iglesias \& Rogers 1996) or the OP (Badnell et al. 2005) project. In both cases, these opacities are combined for low temperatures to opacities from Ferguson et al. (2005). The effects of thermal conductivity have been taken into account following Potekhin et al. (1999) and Cassisi et al. (2007).

Nuclear reaction rates for the pp chain come from the review of Adelberger et al. (2011), except for the ${ }^{7} \mathrm{Li}(p, \alpha){ }^{4} \mathrm{He}$ reaction, which comes from the NACRE II compilation (Xu et al. 2013).

Convection is treated using the mixing length theory (MLT). For UCDs, we generally set $\alpha_{\mathrm{MLT}}$ (the ratio between the mixing length and the pressure scale height) to $\sim 2.0$, the value adopted in BT-Settl model atmospheres for such stars (BHAC15). AR16 model atmospheres also follow the MLT formalism, with $\alpha_{\mathrm{MLT}}=1.5$, which we adopt for the interior when using these models for surface boundary condition. In Section 2.3 we examine the effect of $\alpha_{\mathrm{MLT}}$ on the evolution of UCDs.

\footnotetext{
See http://freeeos.sourceforge.net/ by A. Irwin.
} 


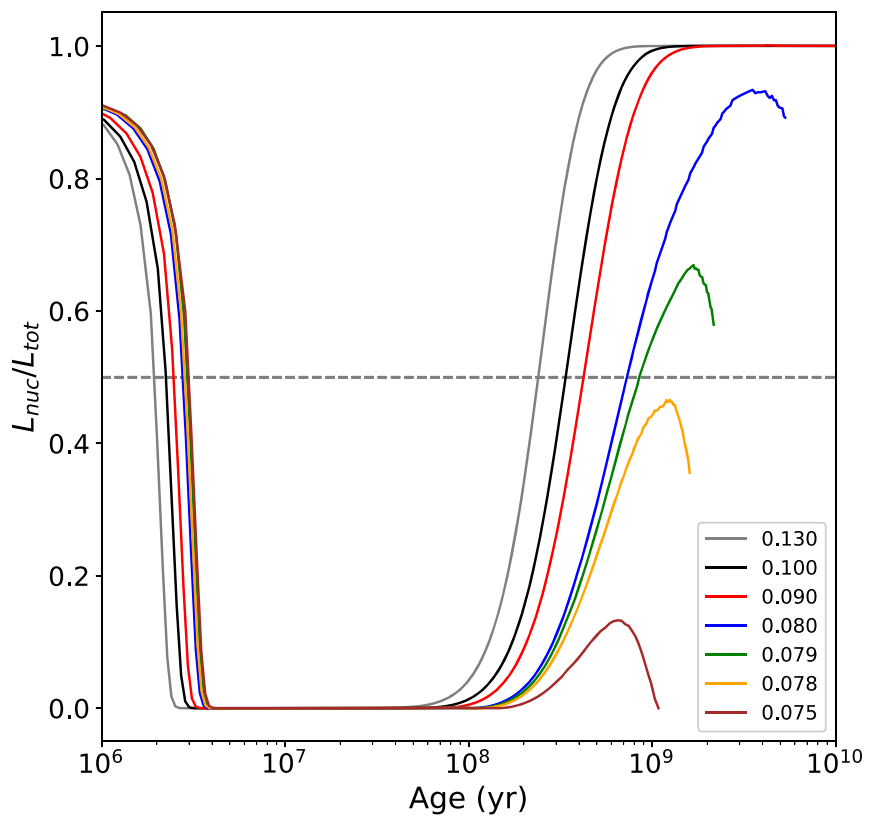

Figure 1. Evolution of fractional luminosity contributed by nuclear fusion reactions $L_{\text {nuc }} / L_{\text {total }}$ for UCD CLES models, showing the tracks between 0.130 and $0.075 M_{\odot}$ assuming solar composition. The dashed line marks the $50 \%$ limit set in Reid \& Hawley (2005) for formally defining $M_{\mathrm{HBL}}$, which in CLES is slightly above $0.078 M_{\odot}$.

CLES solar calibration (evolutionary track giving the Sun luminosity and effective temperature at its present age), without diffusion, with OPAL opacities, with our standard $\mathrm{H}+\mathrm{He}$ $+\mathrm{C}+\mathrm{O}$ EOS, and BCs from BT-Settl model atmospheres, gives $\alpha_{\mathrm{MLT}}=1.8, X_{0}=0.729$, and $Z_{0}=0.013$. This is our standard CLES configuration for UCDs (with $\alpha_{\mathrm{MLT}}=2.0$ ). We note that this solar calibration does not depend highly on the chosen input physics, that is, model atmospheres, EOSs, solar abundances Asplund et al. (2009)/Caffau et al. (2011), and opacities.

\subsection{Properties of CLES Models}

\subsubsection{H-burning Limit Mass}

The formal H-burning limit mass $\left(M_{\mathrm{HBL}}\right)$, i.e., the mass where the fractional contribution to the total luminosity due to hydrogen fusion $L_{\text {nuc }} / L_{\text {total }}$ never exceeds $50 \%$ (Reid \& Hawley 2005), is slightly above $0.078 \mathrm{M}_{\odot}$ in standard CLES configuration (Figure 1). $M_{\mathrm{HBL}}$ is about $0.07 M_{\odot}$ in the BHAC15 models according to their public grids, and $0.073 M_{\odot}$ for Burrows et al. $(1993,1997)$ models. Figure 1 shows that stars with mass slightly higher than $0.08 \mathrm{M}_{\odot}$ achieve stable luminosities and temperatures for many Hubble times, while $0.080 M_{\odot}$ and $0.079 M_{\odot}$ objects are transition objects, able to sustain fusion for several hundreds of million years, but eventually cooling degenerately as BDs. The region where $L_{\text {nuc }} / L_{\text {total }}>0.5$ at early ages for all masses in Figure 1 corresponds to the short-lived phase of D-burning. The $M_{\mathrm{HBL}}$ depends on the chemical composition of the star such that the mass limit increases as metallicity decreases (see also Section 3.4 in Reid \& Hawley 2005). For example, in the CLES models the $M_{\mathrm{HBL}}$ is $0.074,0.078$, and $0.080 M_{\odot}$ at $[M / H]=+0.5$, +0.0 , and -0.3 , respectively.

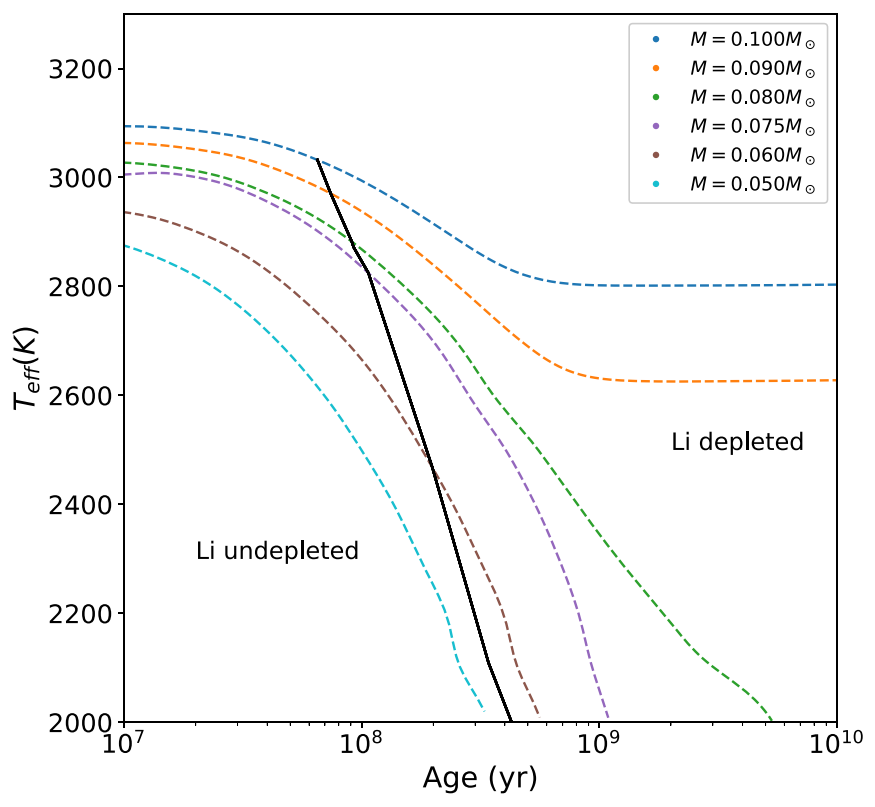

Figure 2. Evolution of effective temperature in CLES models, showing the tracks from 0.100 to $0.050 M_{\odot}$ assuming solar composition. Models to the right of the solid line have depleted lithium by over $90 \%$ from its initial abundance.

\subsubsection{Abundance of Light Elements}

The fusion of light elements, namely $\mathrm{Li}, \mathrm{Be}$, and $\mathrm{B}$, can occur in the interior of UCDs depending on their mass and age. The presence/absence of light elements in the spectra provides a powerful diagnostic to distinguish UCDs of various ages and masses, including BDs (Chabrier \& Baraffe 1997). For example, observing the lithium doublet at $\lambda \mathrm{LiI}=6708 \AA$ was proposed by Rebolo et al. (1992) as the famous lithium test that confirmed the first BD candidates (Rebolo et al. 1995; Basri et al. 1996).

We computed the minimum burning mass for ${ }^{7} \mathrm{Li},{ }^{9} \mathrm{Be}$, and ${ }^{10} \mathrm{~B}$ in CLES models to be $0.053 M_{\odot}, 0.065 M_{\odot}$ and $0.079 M_{\odot}$, respectively, in agreement with literature values, e.g., $0.055 M_{\odot}$, $0.065 M_{\odot}$ and $0.08 \mathrm{M}_{\odot}$, respectively, for Chabrier \& Baraffe (1997), about $0.055 M_{\odot}, 0.07 M_{\odot}$, and $0.09 \mathrm{M}_{\odot}$, respectively, for Burrows et al. (1997). Figure 2 shows the boundary for lithium depletion for when $90 \%$ of lithium has been consumed for different masses.

\subsection{Systematic Error in Evolutionary Models}

\subsubsection{The Choice of EOS}

In our standard EOS, $\mathrm{C}$ and $\mathrm{O}$ are proxies for all metals, with the same C/O proportion as in Asplund et al. (2009). BHAC15 (and older-generation) models gather all metals, including $\mathrm{C}$ and $\mathrm{O}$, in an increased fraction of He. ${ }^{9}$ This is only valid when ionic pressure is negligible compared to electronic pressure, which is true through most of a UCD interior (Chabrier \& Baraffe 1997). Figure 3 quantifies this effect for the first time. We compare stellar luminosity and effective temperature as a function of age, at solar composition, for $0.075 \mathrm{M}_{\odot}$ (below $\left.M_{\mathrm{HBL}}\right), 0.08 \mathrm{M}_{\odot}\left(\right.$ close to $\left.M_{\mathrm{HBL}}\right)$, and $0.09 M_{\odot}\left(\right.$ above $\left.M_{\mathrm{HBL}}\right)$ stars with the CLES standard configuration $\mathrm{EOS}(\mathrm{H}+\mathrm{He}+\mathrm{C}$ $+\mathrm{O}$; solid lines) and the EOS used in the BHAC15 models

\footnotetext{
9 The mixture of H EOS and He EOS is also handled by the additive volume prescription in BHAC15 and older-generation models.
} 

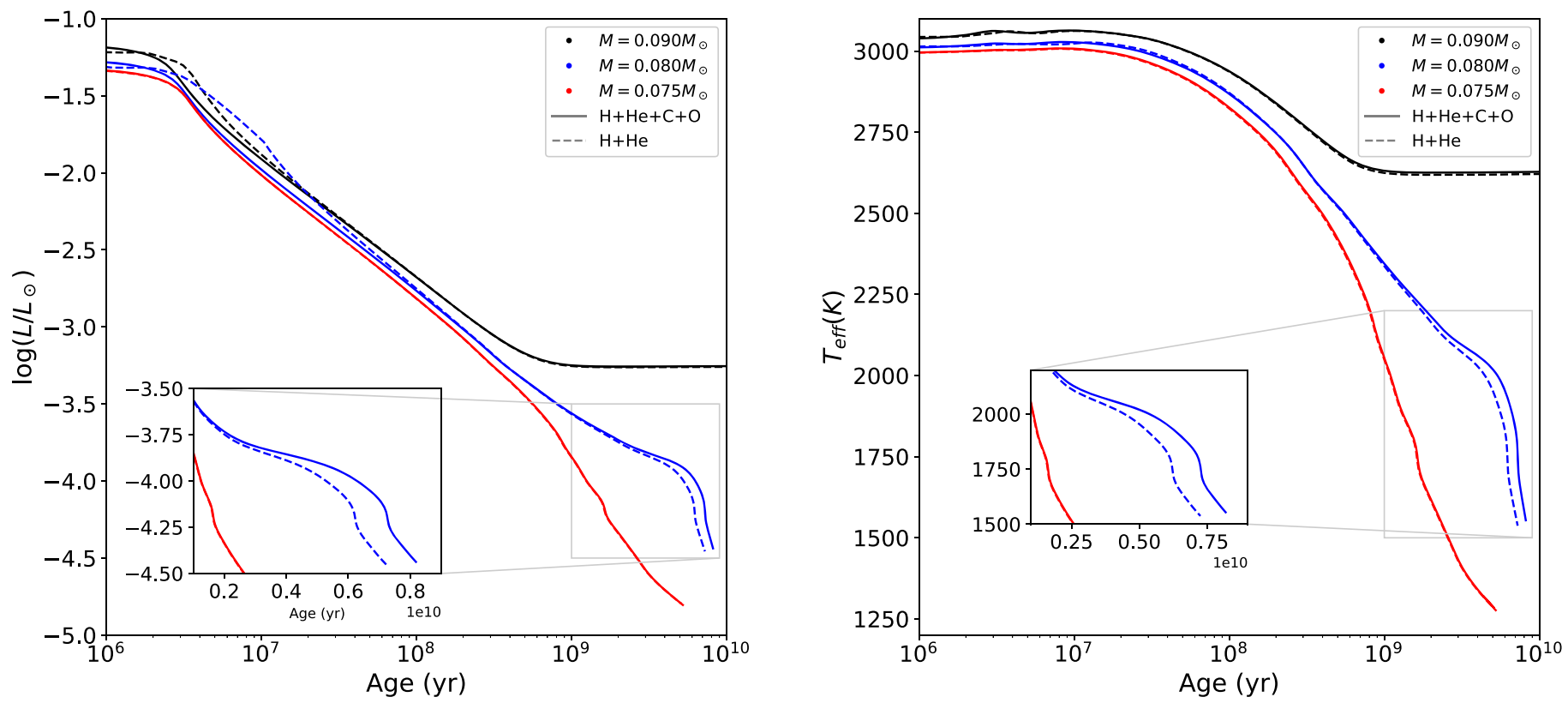

Figure 3. Updated plot stellar luminosity (left) and effective temperature (right) as a function of age for $0.075 M_{\odot}$ (below $M_{\mathrm{HBL}}$ ), $0.08 M_{\odot}$ (at $M_{\mathrm{HBL}}$ ), and $0.09 M_{\odot}$ stars at solar composition, comparing CLES standard EOS (solid line) and $\mathrm{H}+\mathrm{He}$ EOS where metals have been assimilated to helium (dashed line). Close to (far from) $M_{\mathrm{HBL}}$, differences in luminosity and effective temperature are about $4 \%(1 \%)$ and $1 \%(0.2 \%)$, respectively.
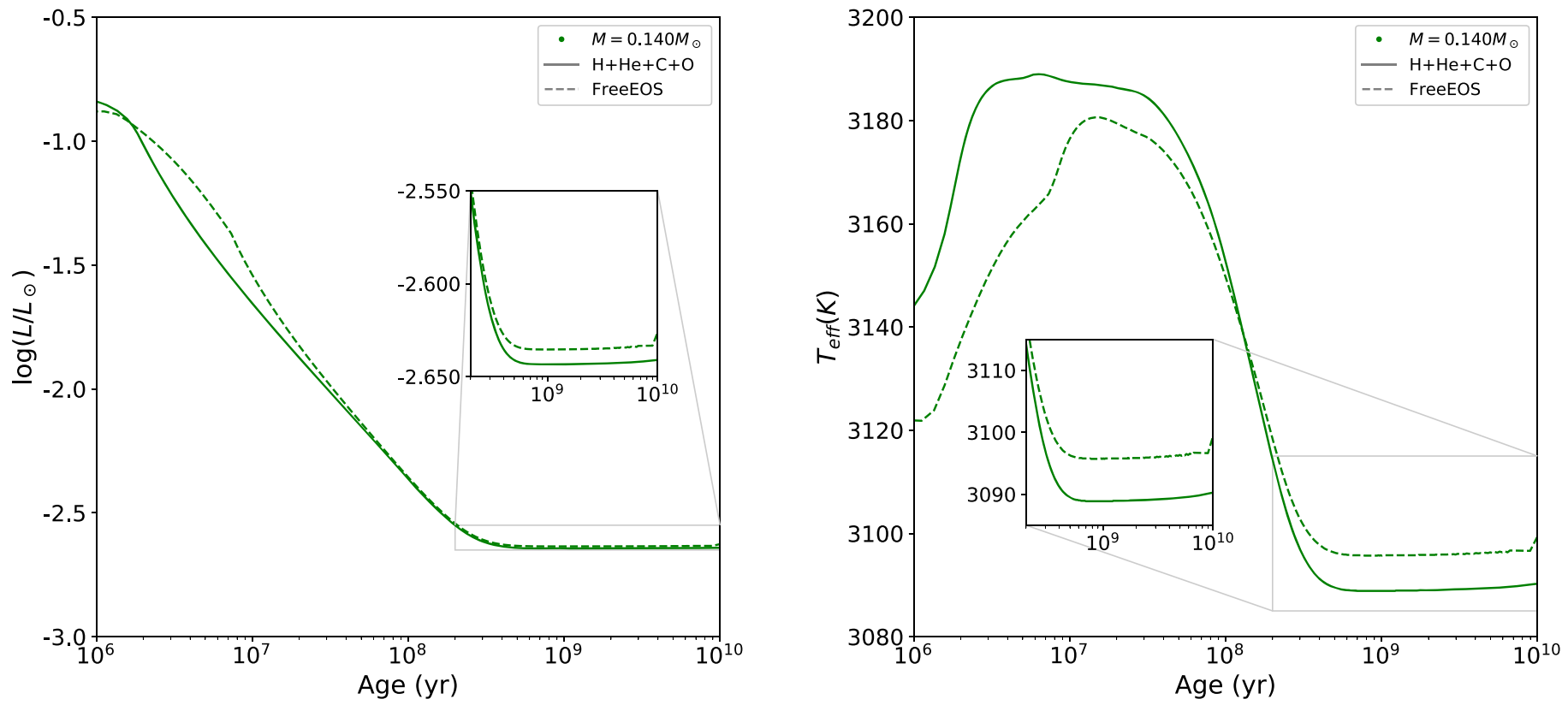

Figure 4. Stellar luminosity (left) and effective temperature (right) as a function of age for $0.14 M_{\odot}$ stars at solar composition, comparing CLES standard EOSs (solid line) and FreeEOS EOSs (dashed line): there is a difference at canonical age of about $2 \%$ in luminosity and $0.2 \%$ in effective temperature.

$(\mathrm{H}+\mathrm{He}$; dashed lines). Figure 3 shows that including the EOS for $\mathrm{C}$ and $\mathrm{O}$ has a strong effect at and close to $M_{\mathrm{HBL}}$, with diverging evolutionary tracks at $0.08 \mathrm{M}_{\odot}$. Close to the $M_{\mathrm{HBL}}$ ( 0.079 and $\left.0.081 M_{\odot}\right)$, the effect on the luminosity and effective temperature is $+4 \%$ and $+1 \%$, respectively. Farther from $M_{\mathrm{HBL}}\left(0.09 \mathrm{M}_{\odot}\right.$ and higher masses; $0.075 \mathrm{M}_{\odot}$ and lower), the impact is smaller, about $+1 \%$ in luminosity and $+0.3 \%$ in effective temperature. The conclusion of this experiment is that assimilating all of the metals into $\mathrm{He}$ is generally a valid hypothesis, but care must be taken close to the $M_{\mathrm{HBL}}$. Some modern stellar evolution codes (e.g., DARTMOUTH, Feiden 2016, and references therein; and PARSEC, Chen et al. 2014), used to model very low-mass stars, provide public grids down to $\sim 0.10 M_{\odot}$, based on the FreeEOS EOS. We found it impossible to make FreeEOS converge for densities/temperatures corresponding to stellar masses below $\sim 0.14 M_{\odot}$. It is plausible that the differences in minimum mass found by different groups comes from choosing different FreeEOS configurations when computing its tables, in particular regarding the numerous ionization states of various elements. In Figure 4, we show the evolution in luminosity of a $0.14 M_{\odot}$ star with FreeEOS and our standard EOS. Differences observed at canonical age are about $2 \%$ in luminosity and $0.2 \%$ in effective temperature. 

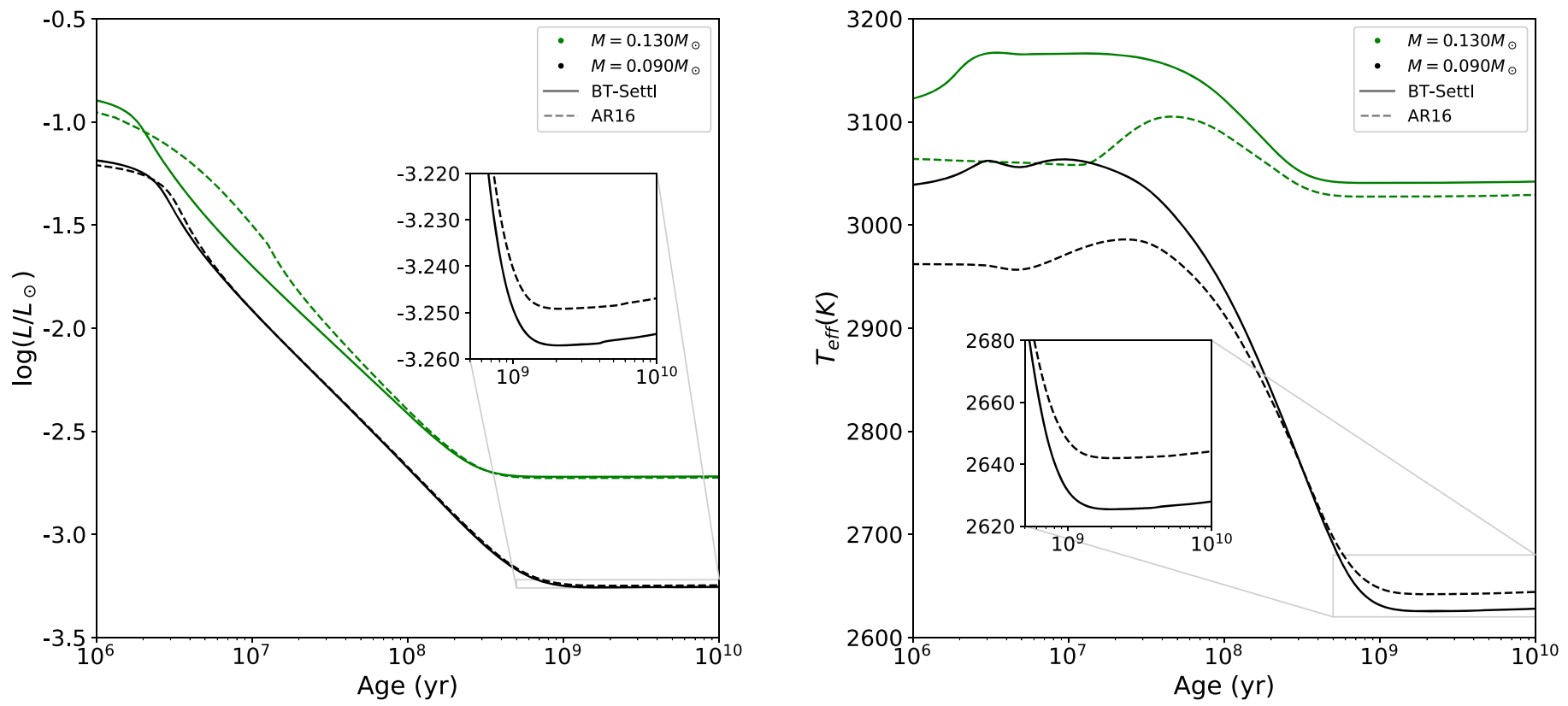

Figure 5. Stellar luminosity (left) and effective temperature (right) as function of age for $0.09 M_{\odot}$ and $0.13 M_{\odot}$ stars at solar composition, comparing BCs extracted from BT-Settl model atmospheres (solid line), and those of AR16 (dashed line). The maximum difference in models with and without grain formation (BT-Settl and AR16, respectively) is about $1 \%-2 \%$ in luminosity and $0.4 \%-0.7 \%$ in effective temperature.

\subsubsection{The Choice of Model Atmospheres as BCs}

Figure 5 compares luminosity and effective temperature as a function of age, at solar composition, for $0.09 M_{\odot}$ and $0.13 M_{\odot}$ with BCs extracted from BT-Settl model atmospheres and AR16 models. Systematic error is small in this case, with maximum differences (in $\mathrm{H}$-burning phase) of $1 \%-2 \%$ in luminosity, $0.4 \%-0.7 \%$ in effective temperature, and $0.2 \%-$ $0.4 \%$ in radius. Models built with BCs based on BT-Settl atmospheres, which include grain formation below $T_{\text {eff }}<2600$ $\mathrm{K}$, are used in a more extended range of stellar mass (including young BDs) than those based on AR16 models, but the latter is more extended in terms of metallicities.

\subsubsection{Other Sources of Systematic Error}

We computed evolutionary tracks with OPAL and OP opacity tables for various masses and various ages at solar composition. OP and OPAL tables give very similar results, i.e., no systematic error is observed by choosing OP rather than OPAL.

We also computed evolutionary tracks with Caughlan \& Fowler (1988) nuclear reaction rates, which were implemented in some older versions of CLES. We found a typical $+1 \%$ increase in luminosity for $0.09 M_{\odot}$ at $10 \mathrm{Gyr}$ using Caughlan \& Fowler (1988) instead of Adelberger et al. (2011).

It is difficult to give estimates linked to the "imperfect" description of convection by the MLT. We note that for UCD objects, the exact value of $\alpha_{\mathrm{MLT}}(1.5,2.0$ or 2.2$)$ has very little influence on model global parameters such as luminosity, radius, and effective temperature, implying that systematic error linked to MLT theory is negligible. Chabrier \& Baraffe (2000) also reported that varying the $\alpha_{\text {MLT }}$ value between 1 and 2 in the interior has no impact for stars below $0.60 M_{\odot}$ (see also Montalbán et al. 2000). We observed the same in our models of UCDs. Only very small values of $\alpha_{\mathrm{MLT}}$ significantly change UCD parameters (see Section 3.2).

\subsubsection{Systematic Error in Models: Conclusions}

In order to provide estimates of the typical systematic error associated with evolutionary models, we carried out a series of experiments reproducing the traditional use of evolutionary models by observers, which consists of inferring a mass from luminosity measurements (determined from parallax and spectral energy distributions typically; see Filippazzo et al. 2015). In order to "translate" systematic error in luminosity by changing input physics (EOS, model atmospheres, nuclear reaction rates) into a systematic error in mass, we identified which shift in mass (for models of a given age) would give a shift in luminosity that is typical for the uncertainty in the measurement of luminosity. We found that all systematic shifts observed in luminosity correspond to a modest change in mass, typically between 0.0001 and $0.0005 M_{\odot}$. We propose that the typical systematic error in mass from evolutionary models, in the range of mass of UCD objects, is $0.0005 M_{\odot}$.

\subsection{Comparison to Existing UCD Models}

The BHAC15 models are commonly used when characterizing UCDs. To compare CLES with BHAC15 models, we have adapted, as far as possible, identical input physics at solar metallicity: Grevesse \& Noels (1993) abundances for the interior models and Asplund et al. (2009) supplemented by Caffau et al. (2011) for some elements for BCs from BT-Settl model atmospheres; EOSs for $\mathrm{H}$ and $\mathrm{He}$ only, with an increased fraction of $\mathrm{He}$ for assimilating metals; identical initial composition (I. Baraffe 2017, private communication); OPAL opacities, no diffusion, $\alpha_{\mathrm{MLT}}=1.6$. A comparison between CLES models and those of BHAC15 with identical input physics has already been presented in Van Grootel et al. (2018) with evolutionary tracks for $0.08,0.09$, and $0.10 M_{\odot}$ stars. Both models have similar luminosity and differences in effective temperature and stellar radius are of about $1 \%$ and $3 \%$, respectively. We complement this study by also including 

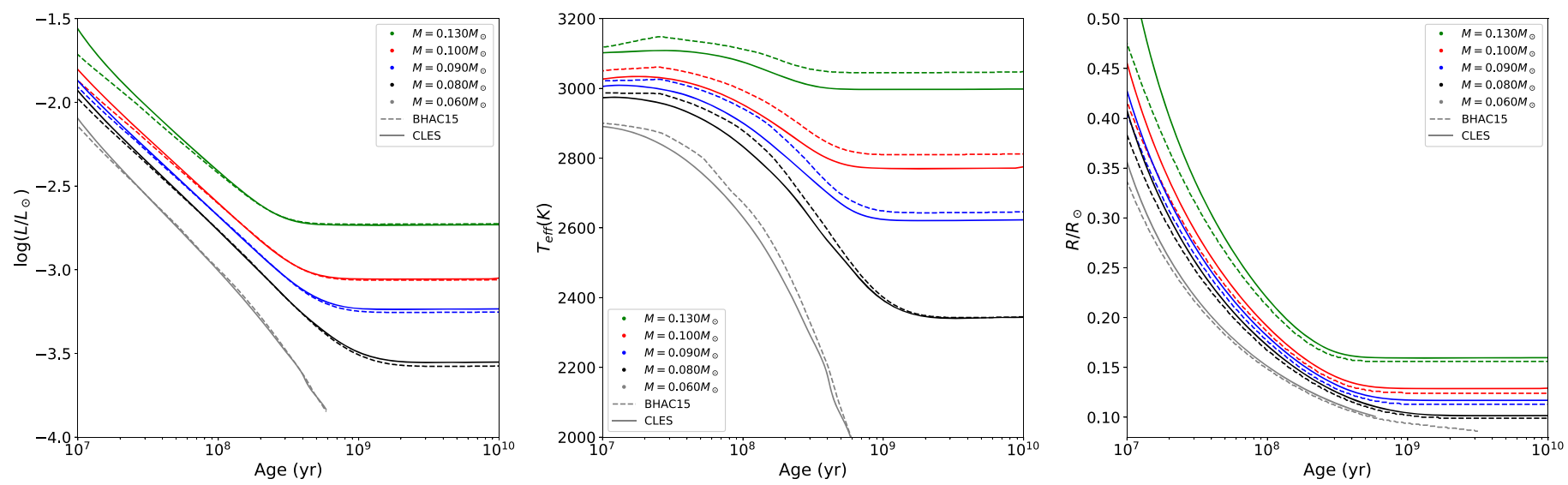

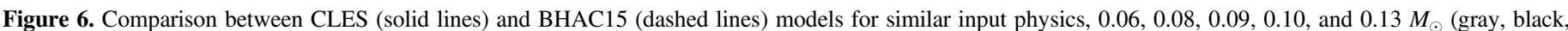

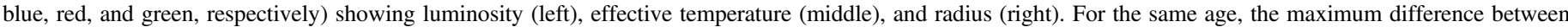
models with similar input physics is of the order of $1 \%-3 \%$.

tracks for 0.130 and $0.06 M_{\odot}$ (the latter below $M_{\mathrm{HBL}}$ ), seen here in Figure 6.

\section{Test Cases}

We test our CLES models by comparing theoretical results with observations for objects for which stellar parameters (luminosity, dynamical mass, age and/or inferred radius from interferometry) have been estimated from independent techniques: the M7 binary LSPM J1314+1320AB (Dupuy et al. 2016, hereafter D16), and the spectroscopic twins GJ 65AB and Proxima Centauri (Kervella et al. 2016, hereafter K16). We also compare these results with those from BHAC15 models.

\section{1. $L S P M J 1314+1320 A B$}

LSPM $\mathrm{J} 1314+1320 \mathrm{AB}$ is a pre-main sequence, nearby binary that has precise measurements of the total dynamical mass and integrated luminosity $\left(M_{\mathrm{tot}}=0.1761 \pm 0.0015 M_{\odot}\right.$ and $\log \left(L_{\text {tot,bol }} / L_{\odot}\right)=-2.322 \pm 0.009$, respectively), as well as the individual masses and luminosities within $\lesssim 1 \%$ precision, obtained through spatially resolved absolute and relative astrometric monitoring and optical and NIR photometry and spectroscopy (D16). The metallicity of LSPM J1314 $+1320 \mathrm{AB}$ has been estimated by D16, using the calibration of Mann et al. (2014), to $[\mathrm{Fe} / \mathrm{H}]=0.04 \pm 0.08$, thus it is consistent with solar metallicity.

In a first test, we used the individual masses and luminosities to model LSPM J1314+1320AB, assuming solar composition and our standard CLES configuration (see Section 2.1). Given the A and B components are nearly equal in mass and luminosity, we took the averaged values $\langle M\rangle=0.0881 \pm 0.0008 M_{\odot}$ and $\left\langle\log \left(L / L_{\odot}\right)\right\rangle=-2.623 \pm 0.010$. Results are presented in Table 1. The quoted errors are from propagating errors on measured mass and luminosity. More precisely, we computed various evolutionary tracks by varying observational constraints $(L, M)$ within their given $1 \sigma$ range and computed the respective $1 \sigma$ confidence interval for output quantities $T_{\text {eff }}$, age, $R$, and $\log g$. Table 1 also shows results obtained with BHAC15 models, as determined by D16, which are similar. Note that, the derived $T_{\text {eff }}$ is about $180 \mathrm{~K}$ hotter than that given by the spectral type- $T_{\text {eff }}$ relation of Herczeg \& Hillenbrand (2014) used by D16, which gives $T_{\text {eff }}=2770 \pm 100 \mathrm{~K}$ for LSPM J1314 A and B.

In a second test, we reproduced the test proposed by D16 that mimicked the typical application of models by observers, i.e., that
Table 1

Stellar Evolutionary Models for LSPM J1314+1320AB

\begin{tabular}{lcc}
\hline \hline & CLES & BHAC15 \\
\hline$T_{\text {eff }}(\mathrm{K})$ & $2950 \pm 6$ & $2950 \pm 4$ \\
age $(\mathrm{Myr})$ & $81.7 \pm 3.6$ & $80.8 \pm 2.5$ \\
$\log g$ & $4.840 \pm 0.013$ & $4.839 \pm 0.009$ \\
$R / R_{\odot}$ & $0.1868 \pm 0.0021$ & $0.1871 \pm 0.0016$ \\
\hline
\end{tabular}

Note. Results from evolutionary models for LSPM J1314+1320AB with reference mean values: $\langle M\rangle=0.0881 \pm 0.0008 M_{\odot}$ and $\left\langle\log \left(L_{\text {bol }} / L_{\odot}\right)\right\rangle=$ $-2.623 \pm 0.010$. Comparison between CLES and BHAC15 models, assuming solar metallicity.

we have access to a measurement of the luminosity and to an effective temperature based on a spectral type $-T_{\text {eff }}$ relation, but not to stellar masses. To carry out this test, we used our in-house Levenberg-Marquadt optimization algorithm (Press et al. 1992, see Van Grootel et al. 2018 for details). We first assumed solar composition and obtained a stellar mass $M=0.049 \pm 0.017 M_{\odot}$ $\left(51 \pm 18 M_{\text {Jup }}\right.$ ) with age $=25 \pm 15 \mathrm{Myr}$ (Figure 7, dashed black curve). Here again, quoted errors simply come from error propagation on $T_{\text {eff }}$ and $L$. No systematic error (see Section 2.3) was included. This result is consistent with the values from D16 interpolated from BHAC15 models: $M=50_{-13}^{+20} M_{\text {Jup }}$ and age $=25_{-17}^{+10} \mathrm{Myr}$, still showing the discrepancy between the mass of stellar models and direct measurements. Adjusting the metallicity of LSPM $\mathrm{J} 1314$ to $[\mathrm{Fe} / \mathrm{H}]=0.12(+1 \sigma)$ yields a higher mass $M=0.057 \pm 0.019 M_{\odot}$, closer to the measured value. To fully reconcile models with observations, we found that we have to increase the metallicity to $+2.5 \sigma$, i.e., $[\mathrm{Fe} / \mathrm{H}]=0.24$ (Figure 7, dotted-dashed green curve). Increasing the metallicity to recover stellar parameters was also found in the study of TRAPPIST-1 (Van Grootel et al. 2018). In the same line, Lindgren \& Heiter (2017) and Rajpurohit et al. (2018a) recently observed a typical average deviation of 0.2 to $0.4 \mathrm{dex}$ in $[\mathrm{Fe} / \mathrm{H}]$ between direct spectral fitting and calibration-based techniques. One possibility is that metallicity is underestimated, and another is residual error in models that can be "adjusted" by artificially increasing metallicity. A third possibility is that the spectral type $-T_{\text {eff }}$ relation depends on the scale in use: for the same spectral type, Rajpurohit et al. (2013), Herczeg \& Hillenbrand (2014), and Passegger et al. (2018) show differences of $10 \%$ in $T_{\text {eff }}$ in the M-dwarf regime. 


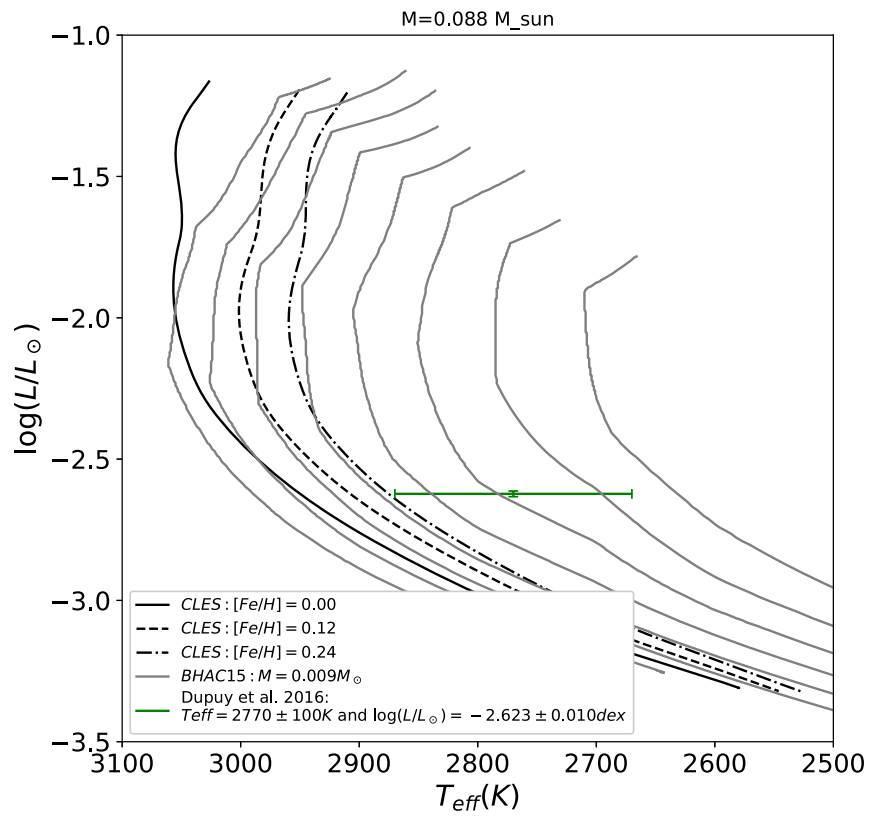

Figure 7. HR diagram comparison between UCD models and reference values for LSPM J1314+1320AB (Dupuy et al. 2016): $T_{\text {eff }}=2770 \pm 100 \mathrm{~K}$ and $\left.\log \left(L_{\text {bol }} / L_{\odot}\right)\right\rangle=-2.623 \pm 0.010$ (green). BHAC15 tracks are from 0.03 to $0.10 M_{\odot}$ by steps of $0.01 M_{\odot}$ and CLES tracks resulted from the optimization process.

\subsection{Proxima Centauri and GJ65 $A B$}

Proxima Centauri (GJ 551, M5.5Ve) is the nearest star after the Sun. It most likely part of a gravitationally bound triple system with $\alpha$ Centauri $\mathrm{AB}$, with an orbital period of about $550000 \mathrm{yr}$ (Kervella et al. 2017). GJ65 AB (BL+UV Ceti, $\mathrm{M} 5.5 \mathrm{Ve}+\mathrm{M} 6 \mathrm{Ve}$ ) is a pair of red dwarfs in a sufficiently large binary to neglect gravitational and magnetic interactions, but sufficiently close to resolve the orbital motion within a human lifetime, with $P_{\text {orb }} \sim 26.3 \mathrm{yr}(\mathrm{K} 16)$. These three nearby stars are among the most studied red dwarfs, and as such constitute cornerstones for models of very low-mass stars. Although they are not strictly speaking UCDs, we can still confront our models with these unique benchmarks.

Proxima and GJ65 AB all have three direct radii measurements from interferometry, respectively $0.141 \pm 0.007 R_{\odot}$ (Demory et al. 2009), $0.165 \pm 0.006 \mathrm{R}_{\odot}$, and $0.159 \pm 0.006 \mathrm{R}_{\odot}(\mathrm{K} 16)$. By orbital modeling, the total mass of GJ65 is accessible, as well as individual masses based on differential astrometry, indicating $0.1225 \pm 0.0043 M_{\odot}$ and $0.1195 \pm 0.0043 M_{\odot}$ for $\mathrm{A}$ and $\mathrm{B}$ components, respectively (K16). No direct mass measurement of Proxima exists, but it can be estimated through mass-absolute magnitude relations, $0.1239 \pm 0.0032 M_{\odot}$ using Mann et al. (2019) at solar metallicity (distance and $K_{S}$ magnitude in Cutri et al. 2003), $0.123 \pm 0.006 \mathrm{M}_{\odot}$ using Delfosse et al. (2000) relations, and $0.118 \pm 0.011 M_{\odot}$ using Henry et al. (1999).

The mass-luminosity relations obtained with CLES models are shown in Figure 8 for solar metallicity, giving us an estimated mass of $0.120 \pm 0.001 M_{\odot}$ for Proxima at $5 \mathrm{Gyr}$ assuming a luminosity $L=0.00155 \pm 0.00002 L_{\odot}$ (Boyajian et al. 2012). Directly determining metallicities from high-resolution spectroscopy of red dwarfs is a delicate task. For Proxima, iron abundance measurements range from $[\mathrm{Fe} / \mathrm{H}]=-0.07 \pm 0.14$ (Passegger et al. 2016) to $[\mathrm{Fe} / \mathrm{H}]=+0.16 \pm 0.20$ (Neves et al. 2014). We could also consider that its membership in the $\alpha$ Cen system indicates a common origin from the same formation cloud, and thus the same age and initial composition. The metallicities of $\alpha$ Cen A and B have been accurately determined from atmospheric abundances of many elements (e.g., Porto de Mello et al. 2008), and stellar modeling and asteroseismology allows us to obtain the initial composition and age, with $X_{0} \sim 0.70, Z_{0} \sim 0.025$ and an age of about $6 \mathrm{Gyr}$ (Bazot et al. 2016). It is also possible that Proxima has been captured by $\alpha$ Cen, and thus does not share a common composition. Whatever the actual metallicity of Proxima, K16 adopted a differential approach to Proxima to determine the metallicity of GJ65 AB. Adopting $[\mathrm{Fe} / \mathrm{H}]=$ $+0.05 \pm 0.20$ for Proxima gave $[\mathrm{Fe} / \mathrm{H}]=-0.03 \pm 0.20$ and $[\mathrm{Fe} / \mathrm{H}]=-0.12 \pm 0.20$ for GJ65 $\mathrm{A}$ and $\mathrm{B}$.

K16 used the BHAC15 models (with solar composition) to model Proxima and GJ65 AB. They found that while Proxima fits with the expected mass-radius relation, GJ65 AB both appear inflated, exceeding model expectations by $14 \% \pm 4 \%$ and $12 \% \pm 4 \%$, respectively. This radius inflation could naturally be explained by a young age (about $250 \mathrm{Myr}$ ), but the GJ65 velocity vector likely indicates a star of at least $1 \mathrm{Gyr}$, and possibly much older. K16 carefully examined possible sources of discrepancy, and concluded that the most likely explanation is a reduced convection efficiency for GJ 65AB by a strong internal magnetic field linked to the relatively fast rotation of both stars $\left(v \sin i \sim 30 \mathrm{~km} \mathrm{~s}^{-1}\right)$. Proxima, on the contrary, is a slow rotator $\left(v \sin i \sim 2 \mathrm{~km} \mathrm{~s}^{-1}\right)$.

We modeled Proxima and GJ65 AB using the same optimization procedure used in the previous section. First, we placed GJ65 AB and Proxima in our CLES mass-radius (M-R) plot with 0.1-5.0 Gyr isochrones, for solar composition (Figure 8). The masses reference for Proxima plotted in Figure 8 are the values from Delfosse et al. (2000) and Henry et al. (1999) (red and orange, respectively), in which we also include our result (blue). We agree with the K16 conclusions: while Proxima reasonably fits the model expectations, GJ65 AB is either a young star (about $300 \mathrm{Myr}$ ), or somewhat inflated compared to its mass. In more detail, we found that models slightly overestimate the stellar radius of Proxima, opposite to the usual trend (see, e.g., Section 3.1 and Van Grootel et al. 2018). The situation would be worse considering Proxima to have the same metallicity as $\alpha$ Cen. We tested the suggestion of K16 to decrease the convection efficiency for GJ65 and found that we had to turn $\alpha_{\text {MLT }}$ down to 0.03 (and even slightly lower for GJ65 A) in order to reconcile the measured radii with model expectations. If we play with metallicity, we have to increase it by a great amount (more than $[\mathrm{Fe} / \mathrm{H}]=+0.5)$ to reconcile interferometric and model radii. This is also unlikely given the differential spectroscopy carried out by K16, which indicates that Proxima and GJ65 have similar metallicities.

We reversed the problem by identifying which stellar mass would fit the radii measured from interferometry, assuming several gigayear stars in both cases. For Proxima we found a mass of $0.113 \pm 0.007 M_{\odot}$. This is within $2 \sigma$ of the value from Delfosse et al. (2000) relations, and within $1 \sigma$ of the Henry et al. (1999) ones. Proxima is at the limit of validity for applicability of such relations $(11,10$, and 9.5 for the $J H K$ absolute magnitudes, respectively, for Delfosse et al. 2000). Indeed, applying such relations for GJ65 (also at the validity limit), K16 found that predicted masses are $12 \%$ and $17 \%$ lower than the measured masses, respectively. Repeating the same exercise with GJ65, we found that we are within $2 \sigma$ of the dynamical value: a mass of $0.137 \pm 0.006 M_{\odot}$ is needed to 

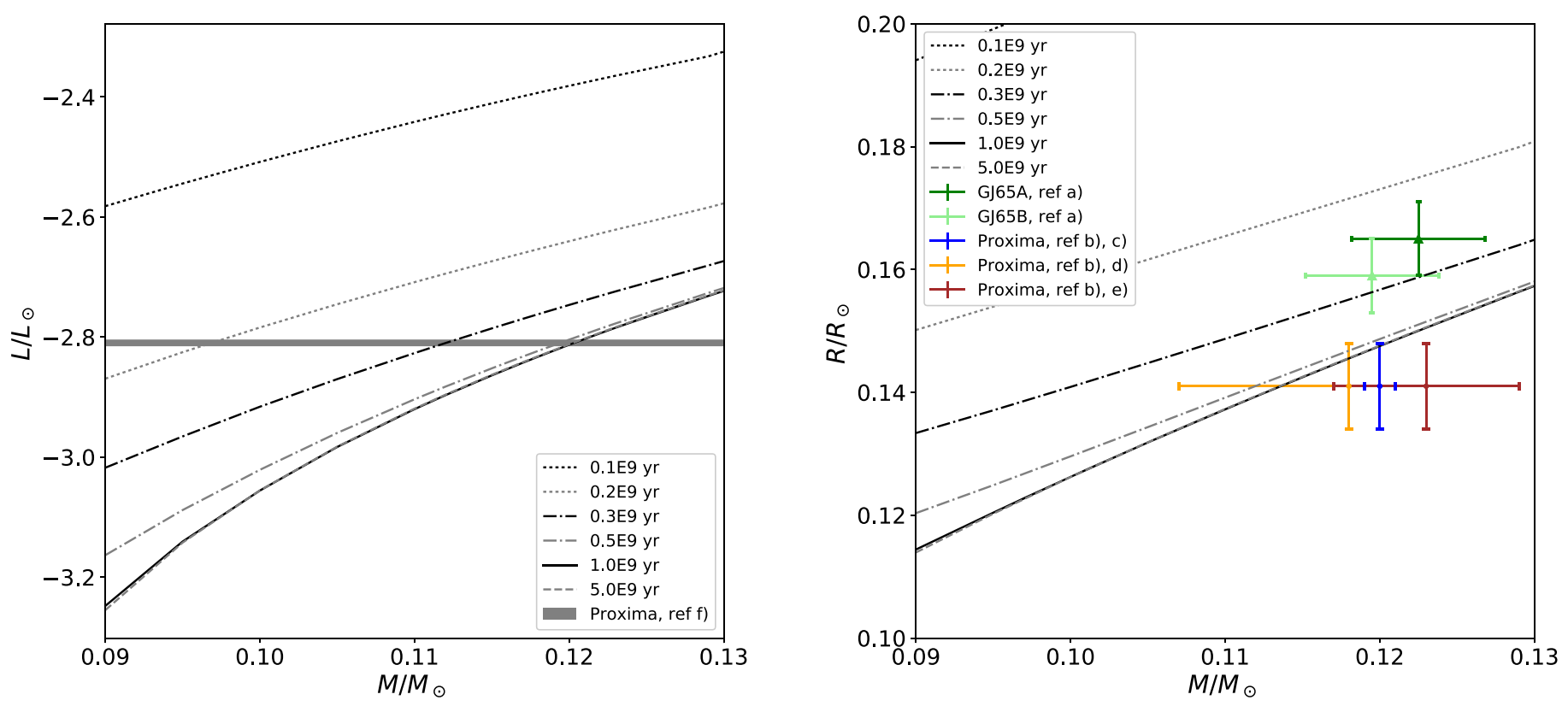

Figure 8. Mass-luminosity (left) and mass-radius relation (right) for CLES models and for several isochrones (0.1-5.0 Gyr), assuming solar composition (see Figure 10 in K16). Compare the to measurements for Proxima and GJ65 AB. References: (a) Kervella et al. (2016), (b) Demory et al. (2009), (c) this work, (d) Henry et al. (1999), (e) Delfosse et al. (2000), (f) Boyajian et al. (2012).

account for the radius of $0.165 \pm 0.006 R_{\odot}$ (GJ 65A), and a mass of $0.132 \pm 0.006 M_{\odot}$ is needed to account for the radius of $0.159 \pm 0.006 R_{\odot}(\mathrm{GJ} 65 \mathrm{~B})$.

In conclusion, given uncertainties in stellar models in metallicity and $\alpha_{\mathrm{MLT}}$ for stars with inhibited convection (e.g., fast rotating or strong magnetic field; see Brun \& Browning 2017), and the uncertainties that are likely to occur in mass-absolute magnitude relations, we posit that an agreement within $2 \sigma$ is acceptable.

\section{Conclusion}

We have presented new evolutionary models for $T_{\text {eff }} \geqslant$ $2000 \mathrm{~K}$ UCD objects, which encompass the very low-mass stars and young, still contracting BDs. These models are based on our in-house evolutionary code CLES, which has been adapted to produce UCD models. In particular, we included a relevant EOS that includes $\mathrm{H}, \mathrm{He}, \mathrm{C}$, and $\mathrm{O}$ elements, and appropriate boundary conditions from two sets of model atmospheres. We presented some properties of our models and investigated their systematic error associated with uncertainties in input physics. We showed a comparison with the reference BHAC15 models in the UCD regime. Finally, a series of test cases was carried out to visualize the strengths and limits of our models.

Tables for our CLES models for various UCD masses and metallicities can be found at http://www.astro.ulg.ac.be/ASTA/ cles-models-UCDs/.

The authors thank the anonymous referee for a helpful review. We warmly thank Didier Saumon for various advice when developing our UCD models. We thank the whole SPECULOOS team (http://www.speculoos.uliege.be/) for motivation, advice, and encouragement while we developed these models. We thank Uffe G. Jørgensen for his comments after carefully reading the manuscript. C.S.F. and S.J.A.J.S. are funded by an Action de Recherche Concertée (ARC) grant financed by the WalloniaBrussels Federation. V.V.G. is F.R.S.-FNRS Research Associate.
The research leading to these results has received funding from the ARC grant for Concerted Research Actions, financed by the Wallonia-Brussels Federation. B.A. was supported by the ERC Consolidator Grant funding scheme (project STARKEY, G.A. no. 615604). G.F. acknowledges the contribution of the Canada Research Chair Program.

Software: CLES (Scuflaire et al. 2008).

\section{ORCID iDs}

Catarina S. Fernandes $(10$ https://orcid.org/0000-00020351-2429

Valérie Van Grootel (1) https://orcid.org/0000-00032144-4316

Adam J. Burgasser (10) https://orcid.org/0000-0002-6523-9536

Gilles Fontaine (1) https://orcid.org/0000-0002-2357-1012

\section{References}

Adelberger, E. G., García, A., Robertson, R. G. H., et al. 2011, RvMP, 83, 195 Allard, F., Homeier, D., \& Freytag, B. 2012a, RSPTA, 370, 2765

Allard, F., Homeier, D., Freytag, B., \& Sharp, C. M. 2012b, MmSAI, 57, 3 Aringer, B. 2000, PhD thesis, Univ. Vienna

Aringer, B., Girardi, L., Nowotny, W., Marigo, P., \& Bressan, A. 2016, MNRAS, 457, 3611

Asplund, M., Grevesse, N., Sauval, A. J., \& Scott, P. 2009, ARA\&A, 47, 481 Badnell, N. R., Bautista, M., Butler, K., et al. 2005, MNRAS, 360, 458 Baraffe, I., Chabrier, G., Allard, F., \& Hauschildt, P. H. 1995, ApJL, 446, L35 Baraffe, I., Chabrier, G., Allard, F., \& Hauschildt, P. H. 1998, A\&A, 337, 403 Baraffe, I., Homeier, D., Allard, F., \& Chabrier, G. 2015, A\&A, 577, A42

Bartlett, J. L., Lurie, J. C., Riedel, A., et al. 2017, AJ, 154, 151

Basri, G., Marcy, G. W., \& Graham, J. R. 1996, ApJ, 458, 600

Bazot, M., Christensen-Dalsgaard, J., Gizon, L., \& Benomar, O. 2016, MNRAS, 460, 1254

Boyajian, T. S., von Braun, K., van Belle, G., et al. 2012, ApJ, 757, 112

Brassard, P., \& Fontaine, G. 1994, in IAU Coll. 147: The Equation of State in Astrophysics, ed. G. Chabrier \& E. Schatzman (Cambridge: Cambridge Univ. Press), 560

Brun, A. S., \& Browning, M. K. 2017, LRSP, 14, 4

Buldgen, G., Reese, D. R., \& Dupret, M. A. 2016, A\&A, 585, A109

Buldgen, G., Salmon, S. J. A. J., Noels, A., et al. 2017a, MNRAS, 472, 751 
Buldgen, G., Salmon, S. J. A. J., Noels, A., et al. 2017b, A\&A, 607, A58 Burrows, A., Hubbard, W. B., Saumon, D., \& Lunine, J. I. 1993, ApJ, 406, 158 Burrows, A., Marley, M., Hubbard, W. B., et al. 1997, ApJ, 491, 856

Caffau, E., Ludwig, H.-G., Steffen, M., Freytag, B., \& Bonifacio, P. 2011, SoPh, 268, 255

Cassisi, S., Potekhin, A. Y., Pietrinferni, A., Catelan, M., \& Salaris, M. 2007, ApJ, 661, 1094

Caughlan, G. R., \& Fowler, W. A. 1988, ADNDT, 40, 283

Chabrier, G., \& Baraffe, I. 1997, A\&A, 327, 1039

Chabrier, G., \& Baraffe, I. 2000, ARA\&A, 38, 337

Chen, Y., Girardi, L., Bressan, A., et al. 2014, MNRAS, 444, 2525

Cutri, R. M., Skrutskie, M. F., van Dyk, S., et al. 2003, yCat, 2, 246

Danel, J.-F., \& Kazandjian, L. 2015, PhRvE, 91, 013103

D’Antona, F., \& Mazzitelli, I. 1996, ApJ, 456, 329

Delfosse, X., Forveille, T., Ségransan, D., et al. 2000, A\&A, 364, 217

Delrez, L., Gillon, M., Queloz, D., et al. 2018, Proc. SPIE, 10700, 107001I

Demory, B.-O., Ségransan, D., Forveille, T., et al. 2009, A\&A, 505, 205

Dupuy, T. J., Forbrich, J., Rizzuto, A., et al. 2016, ApJ, 827, 23

Feiden, G. A. 2016, A\&A, 593, A99

Ferguson, J. W., Alexander, D. R., Allard, F., et al. 2005, ApJ, 623, 585

Filippazzo, J. C., Rice, E. L., Faherty, J., et al. 2015, ApJ, 810, 158

Fontaine, G., Graboske, H. C., Jr., \& van Horn, H. M. 1977, ApJS, 35, 293

Freytag, B., Allard, F., Ludwig, H.-G., Homeier, D., \& Steffen, M. 2010, A\&A, 513, A19

Freytag, B., Steffen, M., Ludwig, H.-G., et al. 2012, JCoPh, 231, 919

Gillon, M. 2017, CHEOPS Fifth Science Workshop 47 (Schloss Seggau, Austria) http://geco.oeaw.ac.at/cheops-workshop-05.html

Gillon, M., Jehin, E., Lederer, S. M., et al. 2016, Natur, 533, 221

Gillon, M., Jehin, E., Magain, P., et al. 2011, EPJWC, 11, 06002

Gillon, M., Triaud, A. H. M. J., Demory, B.-O., et al. 2017, Natur, 542, 456

Grevesse, N., \& Noels, A. 1993, in Origin and Evolution of the Elements, ed.

N. Prantzos, E. Vangioni-Flam, \& M. Casse (Cambridge: Cambridge Univ. Press), 15

Gustafsson, B., Edvardsson, B., Eriksson, K., et al. 2008, A\&A, 486, 951

Henry, T. J., Franz, O. G., Wasserman, L. H., et al. 1999, ApJ, 512, 864

Herczeg, G. J., \& Hillenbrand, L. A. 2014, ApJ, 786, 97

Iglesias, C. A., \& Rogers, F. J. 1996, ApJ, 464, 943

Irwin, A. W. 2012, FreeEOS: Equation of State for stellar interiors calculations, Astrophysics Source Code Library, ascl:1211.002
Kervella, P., Mérand, A., Ledoux, C., Demory, B.-O., \& Le Bouquin, J.-B. 2016, A\&A, 593, A127

Kervella, P., Thévenin, F., \& Lovis, C. 2017, A\&A, 598, L7

Kirkpatrick, J. D., Henry, T. J., \& Simons, D. A. 1995, AJ, 109, 797

Kitsikis, A., Fontaine, G., \& Brassard, P. 2005, in ASP Conf. Ser. 334, 14th European Workshop on White Dwarfs, ed. D. Koester \& S. Moehler (San Francisco, CA: ASP), 65

Lamb, D. Q., Jr. 1974, PhD thesis, The Univ. Rochester

Lindgren, S., \& Heiter, U. 2017, A\&A, 604, A97

Mann, A. W., Deacon, N. R., Gaidos, E., et al. 2014, AJ, 147, 160

Mann, A. W., Dupuy, T., Kraus, A. L., et al. 2019, ApJ, 871, 63

Marley, M. S., \& Leggett, S. K. 2009, ApSSP, 10, 101

Montalbán, J., D’Antona, F., \& Mazzitelli, I. 2000, A\&A, 360, 935

Morley, C. V., Fortney, J. J., Marley, M. S., et al. 2012, ApJ, 756, 172

Neves, V., Bonfils, X., Santos, N. C., et al. 2014, A\&A, 568, A121

Passegger, V. M., Reiners, A., Jeffers, S. V., et al. 2018, A\&A, 615, A6

Passegger, V. M., Wende-von Berg, S., \& Reiners, A. 2016, A\&A, 587, A19

Porto de Mello, G. F., Lyra, W., \& Keller, G. R. 2008, A\&A, 488, 653

Potekhin, A. Y., Baiko, D. A., Haensel, P., \& Yakovlev, D. G. 1999, A\&A, 346,345

Press, W. H., Teukolsky, S. A., Vetterling, W. T., \& Flannery, B. P. 1992 Numerical recipes in FORTRAN (Cambridge: Cambridge Univ. Press)

Rajpurohit, A., Allard, F., Teixeira, G. D. C., et al. 2018a, A\&A, 610, A19

Rajpurohit, A. S., Allard, F., Teixeira, G. D. C., et al. 2018b, A\&A, 610, A19

Rajpurohit, A. S., Reylé, C., Allard, F., et al. 2013, A\&A, 556, A15

Rebolo, R., Martin, E. L., \& Magazzu, A. 1992, ApJL, 389, L83

Rebolo, R., Zapatero Osorio, M. R., \& Martín, E. L. 1995, Natur, 377, 129

Reid, N. I., \& Hawley, S. L. 2005, New Light on Dark Stars: Red Dwarfs, Low-Mass Stars, Brown Dwarfs (Berlin: Springer)

Saumon, D., Chabrier, G., \& van Horn, H. M. 1995, ApJS, 99, 713

Saumon, D., \& Marley, M. S. 2008, ApJ, 689, 1327

Scuflaire, R., Théado, S., Montalbán, J., et al. 2008, Ap\&SS, 316, 83

Tsuji, T., Ohnaka, K., \& Aoki, W. 1996, A\&A, 305, L1

Van Grootel, V., Fernandes, C. S., Gillon, M., et al. 2018, ApJ, 853, 30

Vorberger, J., Tamblyn, I., Militzer, B., \& Bonev, S. A. 2007, PhRvB, 75, 024206

Wang, C., He, X.-T., \& Zhang, P. 2013, PhRvE, 88, 033106

Xu, Y., Takahashi, K., Goriely, S., et al. 2013, NuPhA, 918, 61 